\title{
Cosmogonia*
}

Graziano Pampaloni**

RESUMO: Analisi critica, linguistica e tematica della poesia di P. Levi Nel principio: una cosmogonia laica.

Che cos'è l'esistere se il big bang è plausibile? Per la prima volta, sulla base di una teoria scientifica, respingendo qualsiasi riferimento metafisico e negando le tradizioni ebraica e cristiana, 1' annuncio viene fatto da Primo Levi in una poesia, portentosa, del 13 agosto 1970 .

Ecco il testo:

\section{Nel principio}

1. Fratelli umani a cui è lungo un anno

2. Un secolo un venerando traguardo,

3. Affaticati per il vostro pane,

4. Stanchi, iracondi, illusi, malati, persi;

5. Udite, e vi sia consolazione e scherno:

6. Venti miliardi d'anni prima d'ora,

7. Splendido, librato nello spazio e nel tempo,

8. Era un globo di fiamma, solitario, eterno,

* Recebido para publicação em abril de 1999.

**Lavora presso il dipartimento di italiano dell'UFBA di Salvador. 

9. Nostro padre comune e nostro carnefice
10. Ed esplose, ed ogni mutamento prese inizio.
11. Ancora, di quest'una catastrofe rovescia
12. L'eco tenue risuona dagli ultimi confini.
13. Da quell'unico spasimo tutto è nato
14. Lo stesso abisso che ci avvolge e ci sfida,
15. Lo stesso tempo che ci partorisce e travolge,
16. Ogni cosa che ognuno ha pensato,
17. Gli occhi di ogni donna che abbiamo amato,
18. E mille e mille soli, e questa
19. Mano che scrive.

19 versi, tutti piani, con l'esclusione del nono, quasi al centro della composizione, sdrucciolo. Ma questa 'irregolarità' rimanda a una sinalefe con il verso seguente, per cui la dimensione sennantica di 'carnefice' è completata dall'emistichio seguente 'Ed esplose', che ne costituisce l'attivazione cronologica. Il metro insiste prevalentemente su versi pari (decasillabi, dodecasillabi e martelliani, cioè settenari doppi) con qualche presenza impari (endecasillabi, tredecasillabi); solamente il distico finale presenta misure inferiori, un novenario e un quinario, ma per via dell'enjambement, risulta, ancora una volta, di quattordici sillabe: un doppio settenario. In prevalenza la prima parte (vv.1-9) è costituita da versi dispari (con esclusione dei vv.1 e 4), la seconda (vv.11-19) da versi pari (con esclusione del v.17 e del distico finale, che però è nell'insieme un verso pari.) C'è quindi una disposizione volutamente contrapposta e ne vedremo il senso.

I periodi sono solo tre: il primo di dieci versi (vv.1-10), il secondo di due (vv.11-12), il terzo di sette (vv.13-19); anche ciò è voluto perché corrisponde alla modalità del resoconto scientifico: asserzione, prova, conseguenza. Tuttavia il testo è un'invocazione imperniata sull'Udite del v. 5, per cui le dimensioni della 'convocazione' e della "verità' si mescolano, in quanto la 'voce' raduna per enunciare una 'rivelazione'. Non piú, quindi, qualcosa di lirico e individuale, ma qualcosa di oggettivo e generale, proprio per questo valido per tutti.

Primo Levi è anche il primo poeta che, finalmente, nega eccezionalità e dignità alta alla poesia, per reclamare alla parola, di 
qualsiasi genere e in qualsiasi genere letterario, senza piú gerarchie estetiche, il suo stato, unico possibile, per non essere menzogna, di testimone della verità, della realtà, dei fatti, dei comportamenti, dei dati verificabili e di registrazione memoriale o decifrazione iconica. Basta leggere la prefazione che Levi stesso fece alla raccolta delle sue poesie: In tutte le civiltà, anche in quelle senza scrittura, molti, illustri e oscuri, provano il bisogno di esprimersi in versi, e vi soggiacciono: secernono quindi materia poetica indirizzata a se stessi, al loro prossimo o all'universo, robusta o esangue, eterna o effimera. La poesia è nata certamente prima della prosa. Chi non ha mai scritto versi? Uomo sono. Anch'io, ad intervalli, "ad ora incerta", ho ceduto alla spinta: a quanto pare, è inscritta nel nostro patrimonio genetico. In alcuni momenti, la poesia mi è sembrata più idonea della prosa per trasmettere un'idea o un'immagine. Non so dire perché, e non me ne sono mai preoccupato: conosco male le teorie della poetica, leggo poca poesia altrui, non credo alla sacertà dell'arte, e neppure credo che questi miei versi siano eccellenti. Posso solo assicurare l'eventuale lettore che in rari istanti (in media, non piu di una volta all'anno) singoli stimoli hanno assunto naturaliter una certa forma, che la mia metà razionale continua a considerare innaturale.' $^{\prime}$

Lessicalmente il testo si presenta senza difficoltà interpretative; solamente al v.11: 'quest'una catastrofe rovescia', un conglomerato che richiede attenzione proprio perché complica il significato usuale sia per la presenza del latinismo una, cioè unica, del grecismo catastrofe, qui usato, oltre che nel senso di rivolgimento e in quello metaforico di disastro, molto probabilmente nell'accezione matematica di 'discontinuità' 2 e dell'aggettivo rovescia, nel significato di negativo, opposto al giusto. Ma il testo, per quanto semplice, richiama tutta una serie di fenomeni scientifici e culturali di grande spessore e da districare.

'Cf. P. Levi, Ad ora incerta, Milano, Garzanti, 1990, (1 ed 1984), p.7.

${ }^{2} \mathrm{La}$ teoria delle catastrofi di R. Thom è proprio degli anni sessanta. 
Il titolo stesso presenta una novità polemica nei confronti della tradizione, perché usa la preposizione articolata nel che marca una certezza, una determinazione: c'è un solo inizio, unico e assoluto. Netta è la contrapposizione alla prima parola della Genesi: bereshit, che la traduzione greca detta dei Settanta rese con 'Ev ' $\alpha \rho \chi \eta$, che la Vetus Latina rese con: 'In principio' e che da sempre viene tradotta con 'In príncipio', a indicare semplicemente un inizio narrativo, come d'altra parte si deduce dalla funzione di titolo per l'intera opera, che la prima frase della Genesi svolge. È pur vero che la tradizione cristiana ne ha fatto il topos dell'origine. Infatti il primo sintagma del Vangelo di Giovanni è 'Ev ' $\alpha \rho \chi \eta$, che diventa 'In principio' nella Vulgata e tale rimane anche nella traduzione italiana. Levi, invece, sottilmente fa una distinzione fra l'opzione della tradizione religio$\mathrm{sa}$, che è in un tempo e in una sequenza, e l'origine del tutto. C'è un solo inizio ed è quello cosmologico, collocato, anche temporalmente, in un punto preciso: 'Venti miliardi d'anni prima d'ora' (v.6).

Lo scrittore, si situa su di un piano comune a quello religioso anche se in contrasto con la gerarchia dell' 'annunciazione' e, pur essendo l'annunciatore, è su un piano di uguaglianza con tutti gli esseri umani, che sono infatti 'fratelli' (v.1) e nel corso del testo è ripetuta questa comunanza: 'nostro' (v.9, ben due volte), 'ci' (vv.1415 , ben tre volte), 'abbiamo' (v.17); nessuna eccezionalità, né differenza dunque: egli è semplicemente colui che annuncia, che informa; ha la semplice funzione di avvertire, ma umanamente è come tutti: c'è quindi una condizione umana che non ammette distinzioni o privilegi e da questa uguaglianza non può derivare che sorte comune e solidarietà. In fondo il 'poeta' non è altro che un messaggero, un divulgatore, un postino, un banditore che, per lo piú, comunica ordini, celebra un evento singolare o mette in guardia da pericoli: 'Udite' (v.5), infatti! Eppure quanto è polemicamente diverso questo invito dallo Shema (= ascolta) della tradizione e fondamento di ogni pregare ebraici. Levi si rivolge a tutti: da chi non giunge ad un anno di vita ('cui è lungo un anno' v.1), a chi compie cent'anni ('venerando traguardo' v.2). Si potrebbe dire dagli infanti ai saggi (ma spesso 
nuovamente incapaci di intendere), cioè ad esseri che stanno fra due limiti che collimano con l'insipienza, l'assenza della parola: i preparlanti, gli straparlanti ed, in mezzo, i parlanti. Ma la caratteristica fondamentale di questi è data dall' essere immersi nella pena ('Affaticati /.../ persi', vv.3-4), in un climax, si potrebbe dire, del dolore, che va dalla fatica alla morte. ${ }^{3}$ Però anche qui c'è un'allusione polemica alla Genesi, quando il verso 3 rifà il passo biblico: 'in sudore vultus tui vesceris pane' (='suderai per mangiare il tuo pane', Gen.3,17,), ma anche richiama quel 'Panem nostrum cotidianum da nobis hodie' (Mt.6,11), introdotto da Levi con il possessivo 'vostro'. tipico degli umani. ${ }^{4}$

Significativo, poi, è il dato che le caratteristiche degli umani siano tutte negative, con l'esclusione della condizione di solidarietà (Fratelli v.1), e che assommino a 7: fratelli, affaticati, stanchi, iracondi. illusi, malati, persi; e si vedrà come questa sequenza numerica ritornerà lungo tutto il testo. D'altra parte in sette giorni avvenne la creazione secondo il mito genesiaco.

Che cosa annuncia il nostro banditore? In sostanza la teoria cosmologica del big bang, ma accanto all'enunciazione del 'grande scoppio', della singolarità, c'è pure una controformulazione che annienta i miti tradizionali in vigore, in particolare ebraico-cristiani, perché ascientifici. Per Levi emerge dunque una verità spiazzante, basata su un nuovo paradigma scientifico e tale da rinnegare tutto il precedente, perché elaborata su dati inoppugnabili. Vediamo come.

E sintomatico che questo 'annuncio' sia 'consolazione e scherno' (v.5), e non è da escludere che Levi, con l'ossimoro, accomuni i due

\footnotetext{
${ }^{3} \mathrm{C}$ ' $\mathrm{e}$ da considerare anche il fatto che 'persi' oltre al significato verbale, ha anche un senso, per quanto arcaico, rimandante a un colore bruno, tendente al rossiccio, che è il materiale costitutivo, il fango, 'de limo terrae' (Gen.2,7), di Adamo sia nella Bibbia che nel Corano. Ma anche il Golem della tradizione qabbalistica è fatto con argilla rossa.

'Qui si trascura il fatto che quel 'cotidianum' per molti codici è 'cpiousion (= soprasostanziale), perché la tradizione ha imposto il primo significato.
} 
termini puntando sul valore culturale del primo, che richiama il genere letterario della consolatio, fatta per aiutare a sopportare e superare il dolore; ma, contemporaneamente, ne carichi il messaggio di dileggio, di disprezzo insultante (anche di beffa ed inganno, secondo l'accezione dantesca, cf. Inf. 23,13-14), proprio perché questa 'rivelazione' non è 'consolatoria', perché non è un'eziologia che spiega l'inesplicabile e carica sull'umanità la responsabilità del male e della pena, la convince e costringe a chinare il capo davanti ai potenti e la immerge nel senso di colpa. Quello che emerge è qualcosa di urtante e sarcastico, una duplicità antitetica connaturata, positivo e negativo intimamente intrecciati, per cui la parola stessa del testo non può che essere amorosa e dolorosa. Ma in quello 'scherno' è concentrata tutta la dimensione provocatoriamente spiazzante della scoperta: l'essere immessi irreparabilmente nella condizione in cui siamo ed essere contemporaneamente consapevoli del fatto che la 'parola' nello stesso momento in cui rivela è anche una illusione, perché incapace di risolvere alcunché. La 'parola' è un semplice suono, con cui si può al massimo comporre una cantilena, anche una poesia. Nessuna parola 'crea', è soltanto una maschera e può soltanto suonare, anche se simile suono può manifestarsi come 'rivelazione' (in fondo una apocalissi, appunto uno svelamento, uno smascheramento di ogni ambiguità, falsità, enigma. Nient'altro che una 'alhqeia: una 'verità' dunque).

Ecco, allora, la rivelazione: tutto è iniziato dallo scoppio di un grumo di energia concentrata. Prima di tutto, del tempo e dello spazio, 'librato' (v.7); in una bellezza abbacinante, 'splendido' (v.7); da sempre presente, 'eterno' (v.8); un nodo incandescente, 'globo di fiamma' (v.8); capace di dare vita, 'padre' (v.9) e morte, 'carnefice' (v.9); ed improvvisamente scoppiò: 'Ed esplose' (v.10). Si direbbe che proprio per queste intrinseche e contradditorie possibilità di creatività e distruttività, un agente, contemporaneamente padre e torturatore, dà inizio al tutto.

Ancora una volta si incontrano sette caratteristiche: infatti il ' $g l o-$ bo' è di fiamma. splendido, librato, solitario, eterno, padre, carnefice. 
Comunque anche qui si può riscontrare un'allusione polemica al ' $\mathrm{Pa}$ dre nostro' (Mt.6,9) della tradizione cristiana, e alla sua funzione provvidenziale, dato che con la duplice insistenza su 'nostro', che unifica, appunto, 'padre' e 'carnefice', si colloca la dimensione biotanatica nella stessa origine. ${ }^{5}$ Eppure proprio dall'esplodere, in fondo dalla 'morte' del globo, scatta ciò che chiamiamo vita. È qui la singolarità, l'inizio (v.10), nella nascita di 'ogni mutamento' (v.10), con l'annullamento del 'globo di fiamma' (v.8). Ancora una volta il sarcasmo considera come il venir meno di una condizione, in apparenza, di perfezione e assolutezza scatena invece creazione, eventi, realtà nuove; ma tutto è soggetto all'annientamento, perché è da una 'morte' che si origina il tutto: 'una catastrofe rovescia' (v.11) e quindi il tutto, figlio della morte, non può che morire.

Levi, proprio perché prima di tutto scienziato, porta la prova dell'esplosione: la 'radiazione di fondo' o costante di Hubble, dovuta allo scorrimento verso il rosso per l'effetto doppler, 'L'eco' (v.12), che dimostra la teoria del big bang, insistendo ancora una volta sulla caratteristica 'genetica' di ciò che si è originato dall'esplosione, la flebilità ('tenue', v.12), quindi anch'essa destinata a scomparire e richiamante perciò la consunzione stessa del cosmo: il big crunch, la concentrazione finale.

Il verso 10 è comunque, ancora una volta, polemico nei confronti della tradizione mistico-filosofica di cui Jonas e Pareyson sono stati gli ultimi esponenti, perché contrappone un esplodere all'implodere divino che le tradizioni esoteriche o la speculazione sul dolore hanno individuato e ipotizzano come condizione della 'libertà' umana e della creazione del cosmo. ${ }^{6}$ In Levi, invece, il dolore è connaturato al manifestarsi del cosmo, dato che l'esplosione è uno 'spasimo' (v.13), per di piú 'unico' (v.13), di modo che quella 'catastrofe rovescia'

\footnotetext{
sOccorre ricordare l'asserzione leviana:"C'è Auschwiz, quindi non può esserci Dio.". In: F. Camon, Autoritratto di Primo Levi, Padova, Nord-Est, 1987, p. 72.

"Cf. H. Jonas, I/ concetto di Dio dopo Aluschwitz. Una voce ebraica, Genova, Il Melangolo, $1989 \mathrm{e} \mathrm{L.} \mathrm{Pareyson,}$ Filosofia della libertà, Genova, Il Melangolo, 1989.
} 
(v.11) è qualcosa che, invece di chiudere un evento, si è ribaltata in un avvio della storia del cosmo, ma concentrando in sé il senso di disastro, di rivolgimento contrario, di calamità, che, paradossalmente, ha dato il via alla vita. Invece di essere uno scioglimento, come era nella tragedia, è all'incontrario una complicazione che innesca una peripezia proprio perché l'opposto di una conclusione e avvia 'ogni mutamento' (v.10) a partire da un evento tremendo che alla propria morte produce il dinamismo che manifesta e sviluppa l'universo.

Inoltre è marcata con insistenza l'unicità, nel duplice senso di assolutezza e totalità da una parte, quello di specificità e individualità dall'altra: 'una' (v.10), 'unico' (v.13), 'ogni' (v.10), 'tutto' (v.13), 'ogni' (vv.16 e 17), 'ognuno' (v.16); si crea cosí una correlàzione uno-tutto che, ancora una volta, sottolinea la complessità e contraddittorietà dell'esistere.

E il testo si chiude elencando il risultato dell'esplosione, da cui si origina 'tutto' (v.13): lo spazio, il tempo, il pensiero, l'amore, gli individui ${ }^{7}$, la stessa mano dello scrittore. Ancora una volta sette aspetti, simbolo della pienezza che va dalla totalità incommensurabile fino all'individualità umana, in una gradatio che ha il suo culmine nella scrittura, apparentemente l'aspetto piú banale del tutto, ma espressione dell'io poetico, l'entità che sa decifrare il mistero.

Dunque, seguendo la metodologia scientifica (teoria, prova, risultato), il testo sembra aver concluso il comunicato, ma altre implicazioni si possono ricavare. Vediamone alcune.

La poesia ha il suo centro fisico e filosofico nel verso 10 , che si conclude con 'inizio', che, di per sé, concentra il duplice statuto di sostantivo e verbo, proprio perché la condizione grammaticale rimanda a quella scientifica, dato che con il grande scoppio è scattato anche il tempo. Infatti se si considerano, in sé, tutte le parole finali di

${ }^{7}$ Opto per la lezione 'soli' (v.18) come esseri solitari, individui innervati di solitudine, che è pure piú in sintonia con il climax e con il senso filosofico, piuttosto di quello di galassie. 
verso si potrà notare come nella prima parte (vv.1-9) vi siano solo sostantivi o aggettivi, pur con l'ambiguità di persi ed elerno; mentre nella seconda parte (vv.11-19) solo verbi, se presi in sé, con l'esclusione di questa (v.18) che, tuttavia, per l'incurvatura del verso, viene risucchiato da 'scrive' (v.19). La prima parte dunque è dominata da elementi atemporali (una condizione di stato), la seconda da elementi cronologici (una condizione di azione), ma questo significa pure che la prima parte è la sezione del globo, la seconda del mutamento, che è anche suono. La prima è il regno dell'astoria, la seconda della storia. La prima dell'esistenziale, dell'acronico, dello statico, del potenziale, dell'aculturale e dell'afasico; la seconda del vitale, dello sviluppo temporale, del dinamico, del compientesi, del culturale e del verbale.

In Daniele al capitolo 5, in particolare ai versetti 5 e 24-28, si racconta che il re Belzazzar, dopo aver bevuto, arrogantemente, da vasi sacri, vede in sogno le dita di una mano scrivere tre parole enigmatiche: Mane, Tekel, Fares. Invano gli intellettuali del regime tentano la spiegazione, che verrà da un prigioniero. (La verità viene sempre dai reietti, dagli esclusi, da chi paga di persona, da chi lotta contro il potere senza appropriarsene, dai rivoluzionari, dunque). Daniele, infatti, dà la spiegazione: il tempo è scaduto, il re è stato pesato e trovato inadeguato, gli verrà sottratto il regno e diviso fra Medi e Persiani.

Anche in Levi c'è una 'Mano che scrive' (v.19).

Si possono allora stabilire alcune relazioni e fare delle ipotesi. Intanto la scrittura è qualcosa che ha a che fare con il campo del mistero, anche dell'onirico e dell'inconscio. Proprio per questo rimanda a una densità problematica in quanto richiede una 'traduzione'; per di piú l'universo della segretezza e della complessità è in relazione col passato, richiede concentrazione per essere sviscerato. La spiegazione dell'enigmatico comporta una vita che sa allontanarsi dal baccano, per poter fare spazio all'intuizione: la capacità di associare elementi separati e impensati. Anche nella poesia la mano 
emana direttamente dalla lontanissima esplosione iniziale, come tutte le realtà, ma proprio per questo ha radici e nervature cosí antiche e lontane, che richiedono un decifratore, uno sciamano che sappia auscultare e leggere quei nervi: interpretare-tradurre quindi; e tanto piú capace di esprimere ciò che è 'tenue', perché di ascendenza lontanissima, a contatto con Seqel (= il silenzio), quanto piú è passato attraverso il dolore dell'umanità, vicino perciò alla scaturigine stessa della pena: la singolarità.

Intanto c'è da considerare che Levi instaura un parallelismo fra l'esplosione e la scrittura, segnato dal dimostrativo: 'quest'una catastrofe' (v.11) e 'questa mano' (vv.18-19). Ambedue rappresentano la fattualità, non tanto un processo intellettuale: come la palla di fuoco esplodendo dà origine al tutto distruggendosi, cosí la scrittura, manifestandosi, svela ciò che era nascosto o impensato, proprio perché 'annientandosi' nella lettura diventa suono, un'eco. Di per sé la mano scrive delle icone che vanno interpretate-tradotte; per questo il poeta è un decifratore, banditore, che annuncia perché ha scoperto una 'verità', che ha la stessa funzione dell'esplodere: avviare un evento e un mutamento, che comporta una nuova visione della vita e della realtà. $\mathrm{Ma}$, contemporaneamente, il testo ci avverte che prima dell'esplosione e prima della scrittura esiste solo l'idecifrabile, il silenzio.

Che cos'è questo silenzio? Questo indicibile e insondabile?

Viene una suggestione da Genesi $(1,2)$ dove si dice: '..tenebrae erant super faciem abyssi,...'; che si potrebbe tradurre: 'il nulla comprimeva il vuoto'. Il termine abisso non è altro che il calco del latino abyssus, dovuto a san Gerolamo come calco, a sua volta, del greco ' $\alpha \beta \cup \sigma \sigma o \zeta$ (= senza fondo), con cui la Settanta aveva tradotto l'ebraico tehom. Si tratta di tutti termini femminili ${ }^{8}$ e alludenti alla profondità incommensurabile, al mistero alla immensità oceanica, qualcosa di grandioso e capace di scatenare l'intervento divino, a

${ }^{8}$ Infatti nelle cosmologie orientali preebraiche rimanda al principio femminile: tyanat. 
volte anche visto come forza tremenda, nefasta e mortale (cf., per es., l'episodio di Noè. in: Gn.7,11); a tal punto che poi divenne sinonimo di luogo infernale, prigione demoniaca. Levi riprende l'allusione mediante il bellissimo 'librato nello spazio e nel tempo' (v.7) e proprio dopo altri 7 versi 'abisso' (v.14) a cui, anche se lo fa nascere dall'esplosione, attribuisce la capacità di sfidare. Ma è qualcosa di muto. La polemica, ancora una volta, è con la tradizione mitografica e religiosa. Non è la parola di Elohim a creare, è un'esplosione, un rumore improvviso. La mano trascrive l'icona di quello scoppio attraverso la scia sonora rimasta negli spazi e quel geroglifico richiede una mente, uno sciamano, che lo trasformi in un linguaggio tale da essere annunciato, proprio perché è un evento 'ricostituito' mediante un residuo: 'L'eco tenue' (v.12). Il mondo, perciò, non è un'emanazione della parola: il logos; (come si afferma in Gv.1,1: 'In principio era il logos'). E porre il rumore di un'esplosione all'inizio è porre l'insensato, ma contemporaneamente è respingere ogni logos, ogni mithos, ogni epos (parole, appunto) come qualcosa di fallace ed ingannevole. Diversamente da Ricoeur, che pensava al mito come ad una spinta per l'umanità a cercare il senso della vita nel trascendente 9 Levi appunta al futuro, al fare, all'operare, all'etico dunque. Per lui tutte le 'parole' che hanno raccontato l'origine del cosmo, proprio perché ascientifiche, sono falsità, maschere e da questo lo scherno che irride tutte le credenze annunciando un senso che non ha ascendenza mitica ma scientifica. E mettendo in guardia contro la fiducia nelle 'parole', che non possono risolvere nulla per quanto concerne la condizione umana, al massimo possono costituire una musica (poesia) che ci accompagna nel tempo che chiamiamo vita. Come un'eco delle nostre relazioni, di cui trascrivono dei balbettii parziali e ripetitivi.

Alla mentalità arcaica incuteva timore il disordine. Tutto quello che non era sistemato, la 'rudis indigestaque moles 'di Ovidio (Met.I,7),

${ }^{2} \mathrm{Cl}$. I miti del principio e della fine, in: P:Ricoeur, Finitudine e colpa, Bologna, II Mulino, 1970, pp.419-621. 
massa informe e confusa, appunto, non era che Kaos, in fondo il nonesistente. Ma per Levi l'abisso, cioè l'impensato, è la potenzialità che si esprime nella sfida, nella provocazione (scherno). Per questo i versi finali (13-19) sono caratterizzati dalla peculiarità della ripetizione di strutture sintattiche, semantiche e ritmiche: 'Lo stesso...che ci...e...' //'Lo stesso ...che ci...e...' (vv.14-15), 'Ogni...che...'// '..ogni ...che...' (vv.16-17) con l'occorrenza in piú del suono 'ogn' nello stesso verso 16 in 'ognuno', il polisindeto 'E...e...e...' (v.18), e, al verso 19, il relativo '..che.', rimarcante i precedenti, ma anche tale da attivare sei coppie antitetiche: uno-tutto, abisso-tempo, affetto-lotta, generazioneannientamento, pensare-amare, sterilità-creatività ${ }^{10}$. Il doppio parallelismo 'avvolge-sfida'// 'partorisce-travolge' dei versi 14-15, rafforzato dalla rima interna-olge e dal chiasmo, infine la rima-ato dei versi $16-17$ sono tutti artifici che hanno la funzione di moltiplicare l'effetto d'eco e quindi di marcare l'aggancio, per quanto residuale, con la singolarità, che ha avviato la paternità, ma anche l'inquietudine della domanda, il tormento del senso e della pena. Cosicché l'ultima parte, attivando la presenza, di per sé asemantica, della risonanza, interroga sul senso dell'origine. Se 'tutto è nato' (v.13) dalla singolarità non ne deriva forse un determinismo? Un vincolo per le possibilità di azione? E la responsabilità umana? E la libertà?

Ancora una volta è nel testo che occorre trovare risposta.

Per 13 versi, se si esclude 'Udite' (v.5), che di per sé ha uno statuto particolare, fàtico piú che semantico, tutti i verbi sono intransitivi, mentre dal 14 alla fine sono tutti transitivi. Caratteristiche del globo: fiammeggiare, essere, esplodere, iniziare, librare, risuonare, nascere. Caratteristiche dell'ecumene: avvolgere, sfidare, partorire, travolgere, pensare, amare, scrivere. Ancora una volta la cifra 7 che ritorna. Una realtà endogena, una entità che è tutta rivolta al suo interno, 'autocontemplantesi', si oppone ad una realtà esogena, che si

\footnotetext{
"Se si preferisce la lezione astronomica, è sempre possibile la coppia: cosmo-individuo.
} 
manifesta in quanto entra in relazione. Alcunché di autocentrato rispetto a qualcosa eterocentrato.

Le uniche parole presenti nella prima parte che si richiamano, fortemente, perché in rima (scherno ed eterno), determinano caratteristiche del globo e alludono a qualcosa di atemporale e di sarcastico, alcunché di disumano. Mentre le uniche parole che nella seconda parte si relazionano strettamente, per via della rima, sono nato, pensato, amato, caratteristiche creaturali, umane, richiamanti il tempo, la relazione, il riflettere. Anche qui una ulteriore differenziazione fra lo status del 'generatore' e quelle del 'generato', che, paradossalmente, possiede peculiarità sconosciute a chi/che cosa ha dato avvio al tutto. Quanto il ' $g l o b o$ ' è chiuso in sé e senza relazioni, tanto il 'mutamento' è solo contatto, relazione, bisogno di altro da sé. Una duplice opposizione, antiteticità perciò. Perché una simile contrapposizione? Che senso ha? Ma, soprattutto, perché nel globo non c'è questo aspetto dell'eros, pur essendovi la generazione?

Apparentemente l'annuncio sembra rivolto a tutti: 'Fratelli umani' (v.1); ma è curioso come al verso 17 si dica 'Gli occhi di ogni donna che abbiamo amato'. L'appello, perciò, riguarda solo gli uomini! Perché solo gli uomini amano? E le donne? É ben vero che il Qohelet afferma che la donna è 'più amara della morte' $(7,27)$, ma forse, anche per questo, il testo offre un'apertura impensata, perché stabilisce una relazione fra 'abisso', 'tempo' e 'donna'. Si pensi ai tratti semantici che rimandano a nascere, partorire, affetto, amore, ma anche a quelli che si connettono con dolore, angustia, morte, nei versi dal 13 al 19. La donna, potenziale portatrice di vita, come l'abisso, 'ci avvolge e ci sfida' (v.14), ma pure come il tempo, 'ci partorisce e travolge' (v.15). Appare evidente come la peculiarità della vicenda dell'esplosione sia l'assenza di relazione. Rimossa perché residuo dell'abisso? Tutto avviene per un processo monogenico e ciò che è assente nell'esplosione è proprio il rapporto bipolare. Esiste 'razionalità' ma manca 'relazionalità'; curiosamente, però, ciò che permane a testimoniare l'evento è un'eco (unico elemento relazionale), come a rivendicare la necessità del superamento del narcisismo, 
dato che comporta distruzione. Tuttavia quello che Levi vuol annunciare è, sicuramente, ancora piú denso, perché da una concezione cosmologica del genere si potrebbe far derivare appunto un determinismo tale da eliminare sia la responsabilità che la libertà, per cui anche Shoah e Porraimos risulterebbero immessi nel processo di deresponsabilizzazione.

La costruzione della poesia, gli artifici messi in atto, la divisione fra i due ambiti, il tessuto semantico, rivelano un senso piú articolato. C'è una netta distinzione fra la condizione del globo prima e dopo la singolarità. Lo stato primario è qualcosa di statico e nel 'silenzio', lo stato posteriore è nel 'rumore', di cui pure rimane un residuo registrabile. Cosí c'è stato un prima, un 'silenzio' della conoscenza e c'è un dopo, la scoperta cosmologica, che si manifesta con un grido (Udite), di cui rimane la registrazione poetica. Come c'è stato un sonno di Adamo, un mutismo dunque, prima dell'esplosione di felicità per la visione di Eva (Gn.2,23)." Ma tutti questi stati acronici, silenzi, regni dell'insondabile, in verità sono un concentrato di elaborazione intensissimo. Adamo prima del 'sonno' passa in rassegna tutti gli animali e dà il nome a ciascuno e il passo genesiaco $(2,20)$ addirittura presenta una triplice iterazione sinonimica di 'tutti': 'cuncta animantia et universa volatilia caeli et omnes bestiae terrae'. Quanto lavorio di ricerca e meditazione la stesura del testo ha richiesto? Quanta complessità la ricerca cosmologica odierna sta mettendo in luce, prima della 'secrezione' da un buco nero di un globo!

È proprio allora nell'abisso che giace un sedimento segreto, enigmatico, ma capace, proprio perché nel muto 'in-pensabile', di avviare la singolarità. Come pure la scoperta dell'altro da sé segue sempre un grumo di 'silenzio' che è un'interrogazione.

Nella linea globo, esplosione, mutamento (isolamento) c'è una condizione arelazionale, autocentrata, che comporta determinismo,

\footnotetext{
"Sull'influenza del linguaggio biblico nella poesia leviana, vedasi: I. Rosato, Primo Levi: Ad ora incerta in: Autografo, II (1985) n.5, pp.95-99; ora in: Primo Levi: un'antologia della critica, (a cura di E.Ferrero), Torino, Einaudi, 1997, pp.367-372.
} 
possesso, delirio di onnipotenza, tortura e in fondo annientamento: è la logica e la razionalità del dominio e della gerarchia semplificatrice. Nella linea abisso, sfida, tempo, (coinvolgimento) c'è una condizione di razionalità relazionale, eterocentrata, che comporta ricerca, tentativo, generosità, creatività, amore, anche fallimento: è la logica dell'olismo e della complessità. Nella prima risiede l'improvviso, nella seconda la lentezza. Dalla prima viene quello che finora è stato, dalla seconda può venire quello che sarà.

Se questo universo dipende, secondo la tradizione mitografica, dalla sfida fra l'abisso ed Elohim, ed è quello che conosciamo, proprio perché scrivere è un fare, è dall'abisso che ancora ci sfida, che siamo chiamati, secondo Levi, a costruire un mondo altro.

RESUMO: Análise crítica, lingüistica e temática da poesia de P. Levi Nel Principio: una cosmogonia laica. 